\title{
Abelian profinite groups
}

\author{
by \\ Krzysztof Krupiński (Wrocław)
}

\begin{abstract}
We investigate abelian profinite groups as profinite structures in the sense of Newelski. We show that each abelian profinite group of finite exponent, which is the inverse limit of a system indexed by $\omega$ and considered with the standard structural group, is small, $m$-normal and $m$-stable. We also obtain similar results for abelian profinite groups with some non-standard structural groups.
\end{abstract}

0. Introduction. We start from the general definition of profinite structure. A profinite space is an inverse limit of finite discrete spaces (so the topology is induced from the product of finite spaces of the inverse system). We assume that all inverse systems are countable.

DEFINITION 0.1. A profinite structure is a pair $\left(X, \operatorname{Aut}^{*}(X)\right)$ consisting of a profinite topological space $X$ and a distinguished structural group $\operatorname{Aut}^{*}(X)$ which is a closed subgroup of the group of all homeomorphisms of $X$ respecting the inverse system defining $X$. Two profinite structures $\left(X, \operatorname{Aut}^{*}(X)\right)$ and $\left(Y, \operatorname{Aut}^{*}(Y)\right)$ are isomorphic if there is a homeomorphism $f: X \rightarrow Y$ such that the pullback function $f^{*}$ maps $\operatorname{Aut}^{*}(Y)$ onto $\operatorname{Aut}^{*}(X)$.

A profinite group in this context is an inverse limit of finite groups with a structural group preserving the group action. We say that a structural group of a profinite structure [group] $X$ is standard if it is the group of all homeomorphisms [topological automorphisms] of $X$ respecting the appropriate inverse system. When it is clear what the structural group is we just write $X$ instead of $\left(X, \operatorname{Aut}^{*}(X)\right)$.

Without loss of generality we can and do assume that all connecting maps of the inverse system defining $X$ are surjections.

The simplest examples of profinite groups are products of countably many finite groups. Let $X=\prod_{i \leq \omega} X_{i}$ be such a product. We consider it as the

2000 Mathematics Subject Classification: 03C45, $20 \mathrm{E} 18$.

Key words and phrases: small profinite groups, abelian profinite groups, abelian structures.

Research supported by KBN grant 2 P03A 01624. 
inverse limit of finite groups $X\left\lceil n=\prod_{i<n} X_{i}, n>0\right.$, with the natural projections. The standard structural group consists here of those automorphisms of $X$ which induce automorphisms of each $X\lceil n$ for $n>0$. Any closed subgroup of the standard structural group can be chosen as another structural group of $X$.

We say that $X$ is small if for every natural number $n>0$, on the set $X^{n}=X \times \cdots \times X$ there are countably many orbits under the action of Aut $^{*}(X)$. Profinite structures and groups in this sense have been introduced in [N1] and [N2]. Small profinite groups occur naturally in model theory as profinite groups interpretable in small theories [N1]. Newelski has developed the model theory of small profinite structures. Many results from stable model theory have been proven in this context. $m$-normality and $m$-stability play a prominent role in all these considerations.

The only known explicit examples of small profinite groups are products of finite groups [K1]. More precisely, if $X$ is a product of finitely many finite groups and countably many finite abelian groups of bounded exponent with the standard structural group, then $X$ is small. In [K1] examples of small products of finite groups with some non-standard structural groups are also given.

The goal of this paper is to find new classes of examples of small profinite groups. We generalize most of the results obtained for products of finite groups in [K1] to the case of abelian profinite groups. The main result is Theorem 1.9, which says that each abelian profinite group $X$ of finite exponent, which is the inverse limit of a system indexed by $\omega$ and considered with the standard structural group, is small, $m$-normal and $m$-stable. Just as for products of finite groups in [K1], to prove this result, first we find a description of orbits in $X$ under the action of the standard structural group. However, we obtain such a description by means of model theory of abelian structures, which is a different approach from that in [K1].

Here is a good place to recall that each torsion abelian profinite group is isomorphic to a direct product of finite abelian groups (see [RZ, Corollary 4.3.9]) and to explain why this result does not reduce the case of abelian profinite groups (regarded as profinite structures) to the case of products of finite groups considered in [K1]. The point is that the above result yields only an isomorphism of topological groups and not of profinite structures. More precisely, we are going to consider an abelian profinite group $X$ of finite exponent as a profinite structure with the standard structural group $\operatorname{Aut}^{*}(X)$ induced from the inverse system defining $X$. Corollary 4.3.9 from [RZ] tells us that $X \cong G=\prod_{i \in \omega} G_{i}$ for some finite abelian groups $G_{i}$ but, of course, the inverse system defining $X$ need not be isomorphic to the system of all initial subproducts $G\left\lceil n=\prod_{i<n} G_{i}, n>0\right.$, with the natural projections. So there is no reason for $\left(X, \operatorname{Aut}^{*}(X)\right)$ to be isomorphic to $\left(G, \operatorname{Aut}^{*}(G)\right)$, 
where $\operatorname{Aut}^{*}(G)$ is the standard structural group of $G$ (i.e. it is induced by the inverse system $G\lceil n, n>0)$.

Now we present all necessary definitions and basic facts on profinite structures and groups in the sense of Newelski. For the proofs and more details see [N1], [N2] and [Wa].

Let $X$ be a profinite structure, e.g. a profinite group. Let $A \subseteq X$ be finite. By $\operatorname{Aut}^{*}(X / A)$ we denote the set of elements of $\operatorname{Aut}^{*}(X)$ fixing $A$ pointwise. We say that $V \subseteq X$ is $A$-invariant if $f[V]=V$ for every $f \in \operatorname{Aut}^{*}(X / A)$. If $V$ is additionally closed, then we say that $V$ is $A$-definable. For $a \in X^{n}$ and $A \subseteq X$ we define $o(a / A)=\left\{f(a): f \in \operatorname{Aut}^{*}(X / A)\right\}$ (the orbit of $a$ over $A$ ). Let $O_{n}(A)=\left\{o(a / A): a \in X^{n}\right\}$. From now on $A, B, \ldots$ usually denote finite subsets of $X$ and $a, b, \ldots$ denote elements or finite tuples of elements of $X$.

DEFinition 0.2. We say that a profinite structure $X$ is small if $\left|O_{n}(\emptyset)\right|$ $\leq \omega$ for every natural number $n>0$. Equivalently $O_{1}(A)$ is countable for every finite set $A \subseteq X$.

Every profinite structure can be enlarged to $X^{\text {eq }}$ by adding so-called imaginary elements, i.e. elements of the form $a / E$, where $a \in X^{n}$ and $E$ is a $\emptyset$-definable equivalence relation on $X^{n}$. If $X$ is small, then, for every such $E$, $X^{n} / E$ is still a profinite structure, where the structural group is induced by $\operatorname{Aut}^{*}(X)$ acting on $X / E$. For more details see [N2].

We say that a profinite structure $X$ is interpretable in a profinite structure $Y$ if there is a continuous 1-1 mapping $f$ of $X$ onto a set $f(X)$ definable in $Y^{\mathrm{eq}}$ over a finite set $A$ such that the pullback function maps Aut* $(Y / A)$ onto a closed subgroup of $\operatorname{Aut}^{*}(X)$. It is easy to see that any profinite structure interpretable in a small one is also small.

For a finite $A \subseteq X$, we denote by $\operatorname{acl}(A)$ (or acl ${ }^{\mathrm{eq}}(A)$ ) the algebraic closure of $A$, i.e. the set of those elements of $X^{\mathrm{eq}}$ which have finitely many conjugates under $\operatorname{Aut}^{*}(X / A)$. When $A$ is infinite, we define $\operatorname{acl}(A)=\bigcup\left\{\operatorname{acl}\left(A^{\prime}\right)\right.$ : $A^{\prime} \subseteq A$ is finite $\}$.

DEFinition 0.3. The rank $\mathcal{M}$ is the function from the collection of orbits over finite sets to the ordinals together with $\infty$ satisfying

$\mathcal{M}(a / A) \geq \alpha+1 \quad$ iff $\quad$ there is a finite $B \supseteq A$ with $o(a / B)$ nowhere dense

$$
\text { in } o(a / A) \text { and } \mathcal{M}(a / B) \geq \alpha \text {. }
$$

Definition 0.4. A profinite structure $X$ is $m$-stable if every orbit has an ordinal $\mathcal{M}$-rank. Equivalently there is no infinite sequence $A_{1} \subseteq A_{2} \subseteq \ldots$ of finite subsets of $X$ and $a \in X$ such that $o\left(a / A_{i+1}\right)$ is nowhere dense in $o\left(a / A_{i}\right)$ for every $i$. 
DEFinition 0.5. A profinite structure $X$ is $m$-normal if for every finite $a, A \subseteq X$, there is a clopen $U \ni a$ such that $U \cap o(a / A)$ has finitely many conjugates under $\operatorname{Aut}^{*}(X / a)$.

In the above definition we can choose as $U$ a canonical open neighbourhood of $a$, where by a canonical open set in

$$
X=\lim _{\longleftarrow} X_{i} \subseteq \prod X_{i}
$$

we mean a set of elements of $X$ with the $i$ th coordinate fixed ( $i$ is arbitrary). A canonical open set in $X^{n}$ is a product of canonical open sets in $X$.

It is worth noticing that in [N1] and [N2], $m$-normality and $m$-stability have been investigated only under the assumption of smallness. This is because of good model-theoretic properties of these notions under that assumption.

Finally, I would like to thank the anonymous referee for some interesting comments and suggestions.

1. Description of orbits and the main result. This section is organized as follows: first we recall the notion of abelian structure; then, using it, we obtain a description of orbits in abelian profinite groups under the action of the standard structural group; as a conclusion we obtain the main result, Theorem 1.9.

Definition 1.1. An abelian structure $\mathcal{A}=\left(A,+, P_{i}\right)_{i \in I}$ is an abelian group $(A,+)$ expanded by predicates $P_{i}, i \in I$, defining subgroups of $A^{n_{i}}$ for $n_{i} \in \omega$.

If we add to the language function symbols defining homomorphisms from some cartesian powers of $A$ into $A$, then the arising structure can also be regarded as an abelian structure. Namely, each homomorphism $f: A^{n} \rightarrow A$ can be identified with its graph $G_{f}=\left\{(a, f(a)): a \in A^{n}\right\}$, a subgroup of $A^{n+1}$.

Now we list several examples of abelian structures.

1. A pure abelian group $\mathcal{A}=(A,+)$.

2. A pure $R$-module $\mathcal{A}=(A,+, r \cdot)_{r \in R}$ for an arbitrary ring $R$.

3. An $R$-module $\mathcal{A}=\left(A,+, r \cdot, P_{i}\right)_{r \in R, i \in I}$, where each $P_{i}$ is a unary predicate defining a subgroup of $(A,+)$.

(i) Let $X=\prod_{i \in \omega} X_{i}$ be a product of countably many finite abelian groups, and let predicates $P_{n}, n \in \omega$, be defined in the following way:

$$
P_{n}(\eta) \Leftrightarrow \eta\lceil n=(0, \ldots, 0),
$$

where $\eta\lceil n$ denotes the first $n$ coordinates of $\eta \in X$. Then $\mathcal{X}:=$ $\left(X,+, r \cdot, P_{n}\right)_{r \in \mathbb{Z}, n \in \omega}$ is an abelian structure with the property that 
$f \in \operatorname{Aut}^{*}(X) \Leftrightarrow f \in \operatorname{Aut}(\mathcal{X})$, where $\operatorname{Aut}^{*}(X)$ is the standard structural group of $X$. The same is also true when $X$ is a product of finitely many groups.

(ii) Let $X$ be the inverse limit of an inverse system of finite abelian groups $X_{i}, i \in \omega$. Let $f_{i}: X \rightarrow X_{i}$ be the natural projection and $P_{i}=f_{i}^{-1}(0)<X$. Then $\mathcal{X}:=\left(X,+, r \cdot, P_{i}\right)_{r \in \mathbb{Z}, i \in \omega}$ is an abelian structure such that $f \in \operatorname{Aut}^{*}(X) \Leftrightarrow f \in \operatorname{Aut}(\mathcal{X})$, where $\operatorname{Aut}^{*}(X)$ is the standard structural group of $X$. The same is true if $X$ is the inverse limit of a finite system.

Let $\mathcal{A}=\left(A,+, P_{i}, f_{j}\right)_{i \in I, j \in J}$ be an abelian structure (i.e. $P_{i}$ 's are predicates defining subgroups of cartesian powers of $A$ and $f_{j}$ 's are homomorphisms from cartesian powers of $A$ into $A$ ).

DEFINITION 1.2. A formula $\phi(\bar{x})$ (in the language of $\mathcal{A}$ ) is a p.p. formula if it is equivalent modulo $\operatorname{Th}(\mathcal{A})$ to a formula of the form

$$
\exists \bar{y} \bigwedge_{i<k} P_{n_{i}}\left(t_{1}^{i}(\bar{y}, \bar{x}), \ldots, t_{k_{i}}^{i}(\bar{y}, \bar{x})\right),
$$

where $\bar{y}, \bar{x}$ are finite tuples of variables, $k \in \omega, n_{i} \in I \cup\{=\}\left(\mid=P_{=}(a) \Leftrightarrow\right.$ $a=0)$ and $t_{1}^{i}(\bar{y}, \bar{x}), \ldots, t_{k_{i}}^{i}(\bar{y}, \bar{x})$ are terms in the language $\left\{+, f_{j}\right\}_{j \in J}$.

A particular case is when $\mathcal{A}=(A,+, r \cdot)_{r \in R}$ is a pure module over $R$. Then $\phi\left(x_{1}, \ldots, x_{m}\right)$ is a p.p. formula if it is equivalent modulo $\operatorname{Th}(\mathcal{A})$ to a formula of the form

$$
\exists y_{1}, \ldots, y_{k} \bigwedge_{i<k}\left(\sum_{j=1}^{k} a_{i j} \cdot y_{j}+\sum_{j=1}^{m} b_{i j} \cdot x_{j}=0\right),
$$

where $a_{i j}$ and $b_{i j}$. are the scalar multiplication by elements of $R$. The following fact is known as the Baur-Garavaglia-Monk theorem.

FACT 1.3. In any pure module, each formula $\phi(\bar{x})$ is equivalent to a Boolean combination of p.p. formulas.

The proof of the Baur-Garavaglia-Monk theorem ([Ba], [Z]) is by induction on the structure of the formula $\phi(\bar{x})$. It uses B. H. Neumann's lemma on covering a group by a set of cosets and some basic combinatorial tricks. One can check that to prove this theorem it is enough to have it for atomic formulas and to use the following properties of p.p. formulas (it is not important what p.p. formulas look like, we use only their properties). Let $\mathcal{A}=(A,+, \ldots)$ be a model in which we formulate the theorem $((A,+)$ is an abelian group).

(i) P.p. formulas are closed under existential quantification and conjunction.

(ii) Each p.p. formula $\varphi\left(x_{1}, \ldots, x_{m}\right)$ defines a subgroup of $A^{m}$. 
(iii) If $\varphi(x, y)$ is a p.p. formula, then so is $\varphi(0, y)$.

(iv) If $\varphi(x, y)$ is a p.p. formula and $a$ is a tuple from $\mathcal{A}$ of the same length as $y$, then $\varphi(\mathcal{A}, a)=\emptyset$ or $\varphi(\mathcal{A}, a)$ is a coset of $\varphi(\mathcal{A}, 0)$.

We easily see that in any abelian structure p.p. formulas have all these properties, so as a conclusion we obtain the Baur-Garavaglia-Monk theorem for abelian structures.

We should mention that abelian structures were introduced and originally studied by E. Fisher ([F]). The p.p. elimination for abelian structures is mentioned in [We]. In modern model theory abelian stuctures are basic examples of stable structures, more precisely they are 1-based groups ([Bu]).

We start our consideration of abelian profinite groups from the following easy remark.

REMARK 1.4. If an abelian profinite group is small, then it has a finite exponent.

Proof. Let $G$ be a small abelian profinite group. By Proposition 2.4 of [N1] we know that $G$ is locally finite. Hence it is a torsion abelian profinite group. Lemma 4.3.7 of [RZ] tells us that $G$ has a finite exponent.

From now on we deal with abelian profinite groups which are inverse limits of inverse systems

$$
X_{0} \leftarrow X_{1} \leftarrow \cdots
$$

(indexed by $\omega$ ) of finite abelian groups. We will consider such a group $X$ with the standard structural group $\operatorname{Aut}^{*}(X)$.

For an element $\eta \in X, \eta(n) \in X_{n}$ denotes the $n$th coordinate of $\eta$ and for $\eta=\left(\eta_{1}, \ldots, \eta_{k}\right) \in X^{k}$, we denote by $\eta(n)$ the tuple $\left(\eta_{1}(n), \ldots, \eta_{k}(n)\right) \in X_{n}^{k}$. Let $\alpha=\left(\alpha_{1}, \ldots, \alpha_{m}\right) \in X^{m}$ and $A$ be a finite submodule (over $\mathbb{Z}$ ) of $X$.

Lemma 1.5 (Description of orbits). $o(\alpha / A)=U$, where $U$ consists of the elements $\beta \in X^{m}$ such that for all $k \geq 1$, natural numbers $n_{1} \leq \cdots \leq n_{k}$, $\left(a_{i j}\right)_{1 \leq i, j \leq k} \in M_{k \times k}(\mathbb{Z}),\left(b_{i j}\right)_{i \leq k, j \leq m} \in M_{k \times m}(\mathbb{Z})$ and $\left(\eta_{1}, \ldots, \eta_{k}\right) \in \overline{A^{k}}$ we have

$$
\begin{aligned}
&\left(\exists \bar{y} \in X^{k}\right) \bigwedge_{i=1}^{k}\left(\sum_{j=1}^{k} a_{i j} y_{j}\left(n_{i}\right)+\sum_{j=1}^{m} b_{i j} \alpha_{j}\left(n_{i}\right)+\eta_{i}\left(n_{i}\right)=0\right) \\
& \Leftrightarrow\left(\exists \bar{y} \in X^{k}\right) \bigwedge_{i=1}^{k}\left(\sum_{j=1}^{k} a_{i j} y_{j}\left(n_{i}\right)+\sum_{j=1}^{m} b_{i j} \beta_{j}\left(n_{i}\right)+\eta_{i}\left(n_{i}\right)=0\right) .
\end{aligned}
$$

Proof. ( $\subseteq$ ) is obvious.

(つ) First, in Step 1, we will show this inclusion for the variant of the lemma with $X$ being the inverse limit of a finite system $X_{0} \leftarrow \cdots \leftarrow X_{n}$ of finite abelian groups considered with the standard structural group $\operatorname{Aut}^{*}(X)$ (that is, after an obvious identification, $X=X_{n}$ and $\operatorname{Aut}^{*}(X)=\operatorname{Aut}^{*}\left(X_{n}\right)$ is 
the standard structural group of $X_{n}$, which may be smaller than $\operatorname{Aut}\left(X_{n}\right)$ ). Then, in Step 2, we will conclude that the desired inclusion holds in general.

SteP 1. Assume that $X$ is the inverse limit of a finite system $X_{0} \leftarrow$ $\cdots \leftarrow X_{n}$ of finite abelian groups considered with the standard structural group $\operatorname{Aut}^{*}(X)$.

By example 3(ii) from our list of examples of abelian structures we can replace $X$ by a suitable finite abelian structure $\mathcal{X}$. Since $\mathcal{X}$ is finite, we get

$$
\operatorname{tp}(\alpha / A)=\operatorname{tp}(\beta / A) \quad \text { iff } \quad(\exists f \in \operatorname{Aut}(\mathcal{X} / A))(f(\alpha)=\beta) .
$$

So to finish the proof we have to show that the type $\operatorname{tp}(\alpha / A)$ in variables $x_{1}, \ldots, x_{m}$ is determined by the set of those formulas of the form

$$
\left(\exists \bar{y} \in X^{k}\right) \bigwedge_{i=1}^{k} P_{n_{i}}\left(\sum_{j=1}^{k} a_{i j} y_{j}+\sum_{j=1}^{m} b_{i j} x_{j}+\eta_{i}\right),
$$

where $n_{1} \leq \cdots \leq n_{k} \leq n,\left(a_{i j}\right)_{1 \leq i, j \leq k} \in M_{k \times k}(\mathbb{Z}),\left(b_{i j}\right)_{i \leq k, j \leq m} \in M_{k \times m}(\mathbb{Z})$ and $\left(\eta_{1}, \ldots, \eta_{k}\right) \in A^{k}$, which are satisfied by $\alpha$. This is a consequence of the following statement:

Every formula $\varphi\left(x_{1}, \ldots, x_{m}\right)$ is equivalent modulo $\operatorname{Th}(\mathcal{X})$ to a Boolean combination of formulas of the form

$$
\left(\exists \bar{y} \in X^{k}\right) \bigwedge_{i=1}^{k} P_{n_{i}}\left(\sum_{j=1}^{k} a_{i j} y_{j}+\sum_{j=1}^{m} b_{i j} x_{j}\right),
$$

which is exactly the Baur-Garavaglia-Monk theorem applied to $\mathcal{X}$.

StEP 2. Now consider the general situation from the lemma.

Assume $\beta \in U$. We want to find an $f \in \operatorname{Aut}^{*}(X / A)$ mapping $\beta$ to $\alpha$. For each $n, \beta$ satisfies the set of conditions guaranteeing (in view of Step 1) existence of a $g_{n} \in \operatorname{Aut}^{*}\left(X_{n}\right)$ mapping $\beta(n)$ to $\alpha(n)$ and fixing the set $A(n)=\{\eta(n): \eta \in A\}$ pointwise.

Since Aut* $\left(X_{n}\right)$ is the standard structural group of $X_{n}$ regarded as the inverse limit of the system $X_{0} \leftarrow \cdots \leftarrow X_{n}$, each $g_{n}$ determines a sequence of automorphisms $g_{n, k} \in \operatorname{Aut}^{*}\left(X_{k}\right), k \leq n$.

Now we define recursively sequences $f_{n} \in \operatorname{Aut}^{*}\left(X_{n}\right)$ and $I_{n} \subseteq \omega \backslash n$, $n \in \omega$, such that

1. $I_{0} \supseteq I_{1} \supseteq \cdots$ are all infinite,

2. for $k \in I_{n}$, we have $f_{n}=g_{k, n}$.

The construction is straightforward, using the pigeon-hole principle.

We see that the sequence $f_{n}, n \in \omega$, is an automorphism of the system converging to an $f \in \operatorname{Aut}^{*}(X / A)$ such that $f(\beta)=\alpha$. 
The above lemma is too weak to show Theorem 1.9 below. We need a deeper insight in the structure of orbits (as in Lemma 3.1.1 in [K1]).

Assume that $X$ has a finite exponent $e$. For $n \in \omega$, we denote by ${ }^{n} X$ the inverse limit of the system

$$
X_{n} \leftarrow X_{n+1} \leftarrow \cdots
$$

and we consider it with the standard structural group $\operatorname{Aut}_{n}^{*}(X)$ (of course ${ }^{n} X$ is naturally homeomorphic to $X$, so these spaces can be identified). $o^{n}(\cdot)$ denotes an orbit in $\left({ }^{n} X, \operatorname{Aut}_{n}^{*}(X)\right)$.

Let $\alpha=\left(\alpha_{1}, \ldots, \alpha_{m}\right) \in X^{m}$ and $A$ be a finite submodule (over $\mathbb{Z}_{e}:=$ $\mathbb{Z} / e \mathbb{Z})$ of $X$. For $k, l_{1}, \ldots, l_{m} \in \mathbb{Z}_{e}$ and $a \in A$ we define

$$
n_{k, l, a}= \begin{cases}\max \left\{n \in \omega: k \mid\left(\sum_{i=1}^{m} l_{i} \alpha_{i}(n)-a(n)\right)\right\} & \text { when such a maximal } n \text { exists, } \\ -1 & \text { otherwise. }\end{cases}
$$

Let $N_{\alpha, A}=\max \left\{n_{k, l, a}:(k, l, a) \in \mathbb{Z}_{e} \times \mathbb{Z}_{e}^{m} \times A\right\}+1$. Take $n \geq N_{\alpha, A}$.

Lemma 1.6. $o^{n}(\alpha / A \alpha(n))=U$, where $U$ consists of the elements $\beta \in X^{m}$ such that $\beta(n)=\alpha(n)$ and for all $a \in A, k \in \mathbb{Z}_{e}, l_{1}, \ldots, l_{m} \in \mathbb{Z}_{e}$ we have

$$
k\left|\left(\sum_{i=1}^{m} l_{i} \alpha_{i}-a\right) \Rightarrow k\right|\left(\sum_{i=1}^{m} l_{i} \beta_{i}-a\right) .
$$

Proof. ( $\subseteq$ ) is obvious.

() Take a $\beta \in U$. Let $k \geq 1, n \leq n_{1} \leq \cdots \leq n_{k},\left(a_{i j}\right)_{1 \leq i, j \leq k} \in$ $M_{k \times k}\left(\mathbb{Z}_{e}\right),\left(b_{i j}\right)_{i \leq k, j \leq m} \in M_{k \times m}\left(\mathbb{Z}_{e}\right)$ and $\eta=\left(\eta_{1}, \ldots, \eta_{k}\right) \in A^{k}$.

We define the $\mathbb{Z}_{e}$-linear combinations $t_{i}(\bar{y}, \bar{x}, \bar{z}), i=1, \ldots k$, of variables $\bar{x}=\left(x_{1}, \ldots, x_{m}\right), \bar{y}=\left(y_{1}, \ldots, y_{k}\right)$ and $\bar{z}=\left(z_{1}, \ldots, z_{k}\right)$ in the following way:

$$
t_{i}(\bar{y}, \bar{x}, \bar{z})=\sum_{j=1}^{k} a_{i j} y_{j}+\sum_{j=1}^{m} b_{i j} x_{j}+z_{i}
$$

We need the following fact, which can be found for example in [A]. Its proof uses the Euclidean property of $\mathbb{Z}$.

FACT 1. For every matrix $H \in M_{n \times n}(\mathbb{Z})$ there exist invertible matrices $L, R \in M_{n \times n}(\mathbb{Z})$ such that the matrix LHR is diagonal.

Using Fact 1 we find $\mathbb{Z}_{e}$-linear combinations $t_{i}^{\prime}(\bar{x}, \bar{z}), i=1, \ldots, k$, and elements $k_{1}, \ldots, k_{k} \in \mathbb{Z}_{e}$ such that in each $\mathbb{Z}_{e}$-module $M$, for any tuples $s=\left(s_{1}, \ldots, s_{m}\right) \in M^{m}$ and $r=\left(r_{1}, \ldots, r_{k}\right) \in M^{k}$, we have

$$
M \models \exists \bar{y} \bigwedge_{i=1}^{k} t_{i}(\bar{y}, s, r)=0 \Leftrightarrow M=\bigwedge_{i=1}^{k} k_{i} \mid t_{i}^{\prime}(s, r) .
$$


By Lemma 1.5, to finish the proof we need to show that

$$
\begin{aligned}
&\left(\exists \bar{y} \in X^{k}\right)\left(\bigwedge_{i=1}^{k} t_{i}\left(\bar{y}\left(n_{i}\right), \alpha\left(n_{i}\right), \eta\left(n_{i}\right)\right)=0\right) \\
& \Leftrightarrow\left(\exists \bar{y} \in X^{k}\right)\left(\bigwedge_{i=1}^{k} t_{i}\left(\bar{y}\left(n_{i}\right), \beta\left(n_{i}\right), \eta\left(n_{i}\right)\right)=0\right) .
\end{aligned}
$$

$(\Rightarrow)$ Assume that $\bigwedge_{i=1}^{k} t_{i}\left(\bar{y}\left(n_{i}\right), \alpha\left(n_{i}\right), \eta\left(n_{i}\right)\right)=0$ for some $\bar{y} \in X^{k}$. As $n \leq n_{1} \leq \cdots \leq n_{k}$, it follows that

$$
\bigwedge_{i=1}^{k} t_{i}(\bar{y}(n), \alpha(n), \eta(n))=0 .
$$

By the definition of $t_{i}^{\prime}(\bar{x}, \bar{z}), i=1, \ldots, k$, we get $\bigwedge_{i=1}^{k} k_{i} \mid t_{i}^{\prime}(\alpha(n), \eta(n))$, and the choice of $n$ yields $\bigwedge_{i=1}^{k} k_{i} \mid t_{i}^{\prime}(\alpha, \eta)$. Since $\beta \in U$ we see that $\bigwedge_{i=1}^{k} k_{i} \mid t_{i}^{\prime}(\beta, \eta)$. Once again by the definition of $t_{i}^{\prime}(\bar{x}, \bar{z})$, we infer that there is a $\bar{y}^{\prime} \in X^{k}$ such that

$$
\bigwedge_{i=1}^{k} t_{i}\left(\bar{y}^{\prime}, \beta, \eta\right)=0,
$$

in particular $\bigwedge_{i=1}^{k} t_{i}\left(\bar{y}^{\prime}\left(n_{i}\right), \beta\left(n_{i}\right), \eta\left(n_{i}\right)\right)=0$.

$(\Leftarrow)$ Assume that $\bigwedge_{i=1}^{k} t_{i}\left(\bar{y}\left(n_{i}\right), \beta\left(n_{i}\right), \eta\left(n_{i}\right)\right)=0$ for some $\bar{y} \in X^{k}$. Hence $\bigwedge_{i=1}^{k} t_{i}(\bar{y}(n), \beta(n), \eta(n))=0$; but $\alpha(n)=\beta(n)$, so $\bigwedge_{i=1}^{k} t_{i}(\bar{y}(n), \alpha(n), \eta(n))$ $=0$. As in the proof of $(\Rightarrow)$ we get

$$
\bigwedge_{i=1}^{k} k_{i} \mid t_{i}^{\prime}(\alpha, \eta)
$$

By definition of $t_{i}^{\prime}(\bar{x}, \bar{z})$, we conclude that there is a $\bar{y}^{\prime} \in X^{k}$ such that

$$
\bigwedge_{i=1}^{k} t_{i}\left(\bar{y}^{\prime}, \alpha, \eta\right)=0,
$$

in particular $\bigwedge_{i=1}^{k} t_{i}\left(\bar{y}^{\prime}\left(n_{i}\right), \alpha\left(n_{i}\right), \eta\left(n_{i}\right)\right)=0$.

Now we will prove a general remark.

REMARK 1.7. Let $X$ be an arbitrary profinite structure, $\alpha$ be a finite tuple from $X, A \subseteq X$ (or even $A \subseteq X^{\mathrm{eq}}$ ) and $a \in \operatorname{acl}^{\mathrm{eq}}(A)$. Then

(i) $o(\alpha / A)$ is a union of finitely many orbits over Aa.

(ii) $o(\alpha / A a)$ is an open subset of $o(\alpha / A)$.

Proof. (i) Since $o(a / A)$ is finite, it splits over $A \alpha$ into finitely many orbits. That is, there are finitely many $a_{0}, \ldots, a_{n} \in o(a / A)$ such that

$$
o(a / A)=o\left(a_{1} / A \alpha\right) \cup \cdots \cup o\left(a_{n} / A \alpha\right) .
$$


For each $i \leq n$ choose $\alpha_{i} \in o(\alpha / A)$ with $o\left(a \alpha_{i} / A\right)=o\left(a_{i} \alpha / A\right)$. Then for every $\beta \in o(\alpha / A)$ there is an $i \leq n$ such that $o(a \beta / A)=o\left(a_{i} \alpha / A\right)=$ $o\left(a \alpha_{i} / A\right)$, hence $o(\beta / A a)=o\left(\alpha_{i} / A a\right)$. We see that

$$
o(\alpha / A)=o\left(\alpha_{0} / A a\right) \cup \cdots \cup o\left(\alpha_{n} / A a\right) .
$$

(ii) We have

$$
o(\alpha / A)=\bigcup_{f \in \operatorname{Aut}^{*}(X / A)} f[o(\alpha / A a)]=\bigcup_{f \in \operatorname{Aut}^{*}(X / A)} o(f(\alpha) / A f(a)) .
$$

So by (i), the last union has only finitely many distinct summands and one of them must be open in $o(\alpha / A)$. Hence $o(\alpha / A a)$ is open in $o(\alpha / A)$.

Now we come back to our profinite group $X$. Let $n \in \omega, \alpha \in X$ and $A$ be a subset of $X$ (or even of $X^{\mathrm{eq}}$ ).

Corollary 1.8. (i) $o^{n}(\alpha / A \alpha(n))$ is a union of finitely many orbits in $X$ over $A \alpha(n)$.

(ii) $o(\alpha / A \alpha(n))$ is open in $o^{n}(\alpha / A \alpha(n))$.

(iii) $o(\alpha / A)$ is open in $o^{n}(\alpha / A)$.

Proof. Of course (i) and (iii) follow from (ii), so it is enough to prove (ii). First we see that

$$
o^{n}\left(\alpha / A X_{n}\right)=o\left(\alpha / A X_{n}\right) \subseteq o(\alpha / A \alpha(n)) \subseteq o^{n}(\alpha / A \alpha(n))
$$

and $o(\alpha / A \alpha(n))$ is either open or nowhere dense in $o^{n}(\alpha / A \alpha(n))$. Hence it is enough to show that $o^{n}\left(\alpha / A X_{n}\right)$ is open in $o^{n}(\alpha / A \alpha(n))$.

Since $X_{n}$ can be identified with an element of acl ${ }^{\mathrm{eq}}(\emptyset)$, we use Remark 1.7 to complete the proof.

THEOREM 1.9. Assume that $X$ is the inverse limit of a system indexed by $\omega$ of finite abelian groups and $X$ has a finite exponent e. Let $\operatorname{Aut}^{*}(X)$ be the standard structural group of $X$. Then $\left(X, \operatorname{Aut}^{*}(X)\right)$ is small, m-normal and $m$-stable.

Proof. (1) Smallness. Suppose for contradiction that $\left|O_{1}(A)\right|>\omega$ for some finite subset $A$ of $X$, so $\left|O_{1}(A)\right|=2^{\omega}$. We can assume that $A$ is a finite $\mathbb{Z}_{e}$-submodule of $X$.

For $\alpha \in X$, let $n_{\alpha}$ be the number $N_{\alpha, A}$ defined just before Lemma 1.6. There is a natural number $n$ and $2^{\omega}$ elements $\alpha \in X$ from distinct orbits over $A$ for which $n_{\alpha}=n$. Hence there are $\alpha \in X$ and $\alpha_{i} \in X, i \in 2^{\omega}$, such that

(a) $\alpha_{i}(n)=\alpha(n)$ and $n_{\alpha_{i}}=n_{\alpha}=n$ for all $i \in 2^{\omega}$,

(b) $\alpha_{i} \notin o\left(\alpha_{j} / A\right)$ for all $i \neq j$.

Let

$$
S=\left\{\beta \in X: \beta(n)=\alpha(n) \wedge n_{\beta}=n\right\} .
$$

We see that $S$ is invariant under $\operatorname{Aut}^{*}(X / A \alpha(n))$ and $\operatorname{Aut}_{n}^{*}(X / A \alpha(n))$. 
By Lemma 1.6 and Corollary 1.8(i) we obtain respectively:

(i) there are only finitely many orbits on $S$ under the action of $\operatorname{Aut}_{n}^{*}(X / A \alpha(n))$

(ii) for each $\beta \in S$, the orbit $o^{n}(\beta / A \alpha(n))$ is a union of finitely many orbits in $X$ over $A \alpha(n)$.

By (i) and (ii) we conclude that there are only finitely many orbits on $S$ under the action of $\operatorname{Aut}^{*}(X / A \alpha(n))$. This is a contradiction with (a) and (b).

(2) $m$-normality. Suppose for contradiction that $X$ is not $m$-normal and the lack of $m$-normality is witnessed by an $\alpha \in X^{m}$ and a finite submodule $A$ of $X$, i.e. for each open neighbourhood $U$ of $\alpha$, the set $U \cap o(\alpha / A)$ has infinitely many conjugates under $\operatorname{Aut}^{*}(X / \alpha)$. Let $n \in \omega$.

Claim. $\left({ }^{n} X\right.$, Aut $\left._{n}^{*}(X)\right)$ is not $m$-normal and this is also witnessed by $\alpha$ and $A$.

Proof. It is enough to show that for an arbitrarily small canonical open neighbourhood $U$ of $\alpha$, the set $U \cap o^{n}(\alpha / A)$ has infinitely many conjugates under $\operatorname{Aut}_{n}^{*}(X / \alpha)$.

By Corollary 1.8(iii) the orbit $o(\alpha / A)$ is open in $o^{n}(\alpha / A)$. Let $U$ be an arbitrary canonical open neighbourhood of $\alpha$ such that

$$
U \cap o^{n}(\alpha / A)=U \cap o(\alpha / A) .
$$

Since $\operatorname{Aut}^{*}(X / \alpha) \subseteq \operatorname{Aut}_{n}^{*}(X / \alpha)$, the proof is complete.

Now we choose an $n$ for $\alpha$ and $A$ as before Lemma 1.6. By the claim there are $f \in \operatorname{Aut}_{n}^{*}(X / \alpha)$ and $\beta \in o^{n}(\alpha / A \alpha(n))$ such that $f(\beta) \notin o^{n}(\alpha / A \alpha(n))$.

Showing that $f(\beta) \in o^{n}(\alpha / A \alpha(n))$ we will get a contradiction. By Lemma 1.6 it is enough to prove that for all $a \in A, k \in \mathbb{Z}_{e}, l_{1}, \ldots, l_{m} \in \mathbb{Z}_{e}$ we have

$$
k\left|\left(\sum_{i=1}^{m} l_{i} \alpha_{i}-a\right) \Rightarrow k\right|\left(\sum_{i=1}^{m} l_{i} f\left(\beta_{i}\right)-a\right) .
$$

Assume $k \mid\left(\sum_{i=1}^{m} l_{i} \alpha_{i}-a\right)$. Then $k \mid\left(\sum_{i=1}^{m} l_{i} \alpha_{i}-f(a)\right)$, so $k \mid(a-f(a))$. Now $\beta \in o^{n}(\alpha / A \alpha(n))$, so $k \mid\left(\sum_{i=1}^{m} l_{i} \beta_{i}-a\right)$ by Lemma 1.6. Hence

$$
k \mid\left(\sum_{i=1}^{m} l_{i} f\left(\beta_{i}\right)-f(a)\right)
$$

and finally $k \mid\left(\sum_{i=1}^{m} l_{i} f\left(\beta_{i}\right)-a\right)$.

(3) $m$-stability. Suppose for contradiction that there is an infinite sequence $A_{1} \subseteq A_{2} \subseteq \cdots$ of finite subsets of $X$ and $\alpha \in X$ such that $o\left(\alpha / A_{i+1}\right)$ is nowhere dense in $o\left(\alpha / A_{i}\right)$ for every $i$. We can assume that each $A_{i}$ is a submodule of $X$. 
Let $C_{i}=\left\{(k, l) \in \mathbb{Z}_{e}^{2}:\left(\exists a \in A_{i}\right)(k \mid(l \alpha-a))\right\}$. Let $n_{i}$ be the number $N_{\alpha, A_{i}}$ defined before Lemma 1.6, so $n_{1} \leq n_{2} \leq \cdots$.

We know that $o\left(\alpha / A_{i+1} \alpha\left(n_{i+1}\right)\right)$ is nowhere dense in $o\left(\alpha / A_{i} \alpha\left(n_{i+1}\right)\right)$. By Corollary 1.8(ii) we conclude that $o^{n_{i+1}}\left(\alpha / A_{i+1} \alpha\left(n_{i+1}\right)\right)$ is nowhere dense in $o^{n_{i+1}}\left(\alpha / A_{i} \alpha\left(n_{i+1}\right)\right)$.

Using Lemma 1.6 one can conclude that $C_{i} \subsetneq C_{i+1}$ for every $i$. So we get an infinite increasing sequence $C_{1} \subsetneq C_{2} \subsetneq \cdots \subseteq \mathbb{Z}_{e}^{2}$, a contradiction.

Finally, we mention that the description of orbits in products of finite abelian groups, given in [K1, Lemma 3.1.1], can also be obtained by means of model theory of abelian structures. Namely, we can apply Lemma 1.5 (in fact, we use here the Baur-Garavaglia-Monk theorem for abelian structures from example 3(i)) and Fact 1 formulated in the proof of Lemma 1.6 (for the details see [K3, proof of Lemma 2.1.6]). In [K1] we proved this description using a group-theoretic result ([K1, Lemma 2.1]).

For the reader's convenience, we recall here both the group-theoretic result mentioned above and the description of orbits in products of finite abelian groups.

FACT 1.10. Let $X=X_{0} \times X_{1} \times \cdots \times X_{w}$ be a finite product of finite abelian groups, $e$ be the exponent of $X$ and $n \leq w$. If we have finite submodules $A$ and $B$ of $X$, an isomorphism $f$ between them and $\alpha \in X, \beta_{n} \in X\lceil n$ such that

$$
(\forall a \in A)\left(\forall k \in \mathbb{Z}_{e}\right)(\forall m \leq w+1)(k \mid a\lceil m \Leftrightarrow k \mid f(a)\lceil m)
$$

and

$$
\begin{aligned}
(\forall a \in A)\left(\forall k, l \in \mathbb{Z}_{e}\right) & (\forall m \leq n) \\
& \left(k \mid\left(l \alpha \left\lceilm-a\lceil m) \Leftrightarrow k \mid\left(l \beta_{n}\lceil m-f(a)\lceil m)),\right.\right.\right.\right.
\end{aligned}
$$

then there exists a $\beta_{n+1} \in X\left\lceil n+1\right.$ extending $\beta_{n}$ such that

$$
(\forall a \in A)\left(\forall k, l \in \mathbb{Z}_{e}\right)\left(k \mid\left(l \alpha \left\lceiln+1-a\lceil n+1) \Leftrightarrow k \mid\left(l \beta_{n+1}-f(a)\lceil n+1)\right) .\right.\right.\right.
$$

For $n=0$ there is no $\beta_{0}$ and then Fact 1.10 says that there exists a $\beta_{1} \in X_{0}$ satisfying the last condition.

Let $X=\prod_{i \in \omega} X_{i}$, where all $X_{i}$ 's are finite abelian groups, and let the exponent $e$ of $X$ be finite. So $X$ is a module over $\mathbb{Z}_{e}$. Let $\alpha=\left(\alpha_{1}, \ldots, \alpha_{m}\right)$ be a tuple from $X$ and $A$ be a finite submodule of $X$.

FACT 1.11 (Description of orbits). $o(\alpha / A)=U$, where $U$ consists of the elements $\beta \in X^{m}$ such that for all $a \in A, k \in \mathbb{Z}_{e}, l_{1}, \ldots, l_{m} \in \mathbb{Z}_{e}$ and $n \geq 1$ we have

$$
k \mid\left(\sum _ { i = 1 } ^ { m } l _ { i } \alpha _ { i } \left\lceiln-a\lceil n) \Leftrightarrow k \mid\left(\sum_{i=1}^{m} l_{i} \beta_{i}\lceil n-a\lceil n) .\right.\right.\right.
$$


2. Modifications of the structural groups. In this section we will prove several results, similar to Theorem 1.9, for abelian profinite groups with some non-standard structural groups. To do this we need a description of orbits more general than in Lemma 1.6. We will obtain such a description by means of model theory of many-sorted abelian structures. Such structures were introduced by E. Fisher ([F]). The following definition can be found for example in [PRZ].

Definition 2.1. A many-sorted abelian structure $\mathcal{A}=\left(\left(A_{s},+\right)_{s \in S}, P_{i}\right)_{i \in I}$ is a disjoint union of abelian groups $\left(A_{s},+\right)$ (these are sorts of $\mathcal{A}$ ) expanded by predicates $P_{i}, i \in I$, defining subgroups of cartesian products of finitely many sorts.

Just as for one-sorted abelian structures, if we add to the language function symbols defining homomorphisms from cartesian products of finitely many sorts into sorts, then the arising many-sorted structure can also be regarded as a many-sorted abelian structure.

Now we continue our list of examples of abelian structures, this time many-sorted ones.

4. A one-sorted abelian structure.

5. A structure $\mathcal{A}=\left(\left(A_{i},+, r \cdot\right)_{r \in R, i \in \omega}, f_{i j}\right)_{i, j \in \omega}$, whose sorts are pure $R$-modules $\left(A_{i},+, r \cdot\right)_{r \in R}, i \in \omega$, and each $f_{i j}: A_{i} \rightarrow A_{j}$ is a homomorphism of $R$-modules.

(i) Let $\left\{X_{i}\right\}_{i \in \omega}$ be a family of finite abelian groups. Let $X=\prod_{i \in \omega} X_{i}$ and $A_{n}=X\left\lceil n\right.$ for $n \geq 1$. For $n \geq m$ we define $f_{n m}: A_{n} \rightarrow A_{m}$ to be the restriction. Then $\mathcal{A}:=\left(\left(A_{n},+, r \cdot\right)_{r \in \mathbb{Z}, n \in \omega}, f_{n m}\right)_{n \geq m}$ is a many-sorted abelian structure. Let $\operatorname{Aut}^{*}(X)$ be the standard structural group of $X$. Then each $f \in \operatorname{Aut}(\mathcal{A})$ induces an automorphism $\widehat{f} \in \operatorname{Aut}^{*}(X)$. On the other hand, all the restrictions to $A_{n}$ 's of an arbitrary $f \in \operatorname{Aut}^{*}(X)$ form an automorphism of $\mathcal{A}$. The same is also true if there are only finitely many groups $X_{i}$.

(ii) Let $X$ be the inverse limit of an inverse system $\left(X_{i}, f_{i j}\right)_{j \leq i \in \omega}$ of finite abelian groups. Then $\mathcal{A}:=\left(\left(X_{i},+, r \cdot\right)_{r \in \mathbb{Z}, i \in \omega}, f_{i j}\right)_{i \geq j}$ is a many-sorted abelian structure. As always we assume that all $f_{i j}$ 's are surjections. As above we have the natural correspondence between $\operatorname{Aut}(\mathcal{A})$ and the standard structural group $\operatorname{Aut}^{*}(X)$ of $X$.

Let $\mathcal{A}=\left(\left(A_{s},+\right)_{s \in S}, P_{i}, f_{j}\right)_{i \in I, j \in J}$ be a many-sorted abelian structure. In the many-sorted language of $\mathcal{A}$ we consider formulas and we define p.p. formulas in the same way as in Definition 2.1, remembering that each variable ranges over a specified sort. 
As for one-sorted abelian structures, it is easy to check that in each many-sorted abelian structure atomic formulas are p.p. formulas and that p.p. formulas have properties (i), (iii), (iv) (see comments following Fact 1.3) and

(ii) $^{\prime}$ Each p.p. formula $\varphi\left(x_{1}, \ldots, x_{m}\right)$ defines a subgroup of a product of appropriate sorts $A_{s_{1}} \times \cdots \times A_{s_{m}}$.

So the Baur-Garavaglia-Monk theorem holds for many-sorted abelian structures.

We recall that we deal with abelian profinite groups which are inverse limits of inverse systems

$$
X_{0} \leftarrow X_{1} \leftarrow \cdots
$$

(indexed by $\omega$ ) of finite abelian groups, and we consider such a group $X$ with the standard structural group $\operatorname{Aut}^{*}(X)$.

For natural numbers $m \leq n$ and an element $\eta \in X_{n}, \eta(m) \in X_{m}$ denotes the image of $\eta$ under the connecting map $f_{n m}: X_{n} \rightarrow X_{m}$ (for $m>n, \eta(m)$ means nothing and is skipped in any expression below). Let $\alpha=\left(\alpha_{1}, \ldots, \alpha_{m}\right) \in X_{m_{1}} \times \cdots \times X_{m_{m}}$ for some $m_{1}, \ldots, m_{m} \in \omega \cup\{\omega\}$, where $X_{\omega}=X$. Let $A \subseteq \bigcup_{n \in \omega \cup\{\omega\}} X_{n}$. We will consider orbits over $A$ so without loss of generality we can assume that $A$ is closed under connecting maps and under projections from $X$ onto each $X_{n}, n \in \omega$, and for each $n \in \omega \cup\{\omega\}$, $A \cap X_{n}$ is a submodule of $X_{n}$.

LEMma 2.2 (Generalized description of orbits). $o(\alpha / A)=U$, where $U$ consists of the elements $\beta \in X_{m_{1}} \times \cdots \times X_{m_{m}}$ such that for all $k \geq 1$, natural numbers $n_{1} \leq \cdots \leq n_{k},\left(a_{i j}\right)_{1 \leq i, j \leq k} \in M_{k \times k}(\mathbb{Z}),\left(b_{i j}\right)_{i \leq k, j \leq m} \in M_{k \times m}(\mathbb{Z})$ and $\left(\eta_{1}, \ldots, \eta_{k}\right) \in A^{k}$ we have

$$
\begin{aligned}
\left(\exists \bar{y} \in X^{k}\right) & \bigwedge_{i=1}^{k}\left(\sum_{j=1}^{k} a_{i j} y_{j}\left(n_{i}\right)+\sum_{j=1}^{m} b_{i j} \alpha_{j}\left(n_{i}\right)+\eta_{i}\left(n_{i}\right)=0\right) \\
& \Leftrightarrow\left(\exists \bar{y} \in X^{k}\right) \bigwedge_{i=1}^{k}\left(\sum_{j=1}^{k} a_{i j} y_{j}\left(n_{i}\right)+\sum_{j=1}^{m} b_{i j} \beta_{j}\left(n_{i}\right)+\eta_{i}\left(n_{i}\right)=0\right) .
\end{aligned}
$$

Proof. The proof is similar to the proof of Lemma 1.5. This time we use the Baur-Garavaglia-Monk theorem for many sorted abelian structures from example 5(ii) on our list of examples of abelian structures.

Assume now that $X$ has a finite exponent $e$. Let $\alpha=\left(\alpha_{1}, \ldots, \alpha_{m}\right) \in X^{m}$ and $A$ be a finite submodule (over $\mathbb{Z}_{e}$ ) of $X$. Moreover, let $A_{0} \subseteq \bigcup_{n \in \omega \cup\{\omega\}} X_{n}$ be closed under connecting maps and projections from $X$ onto each $X_{n}$, $n \in \omega$, and assume that for each $n \in \omega \cup\{\omega\}, A_{0} \cap X_{n}$ is a submodule of $X_{n}$. For $n \in \omega$, we define $A_{0}^{\geq n}=A_{0} \cap\left(X \cup \bigcup_{i \geq n} X_{i}\right)$. 
For $k, l_{1}, \ldots, l_{m} \in \mathbb{Z}_{e}$ and $a \in A$ we define

$$
n_{k, l, a}=\max \left\{n \in \omega:\left(\exists \eta \in A_{0} \cap X_{n}\right)\left(k \mid\left(\sum_{i=1}^{m} l_{i} \alpha_{i}(n)-a(n)-\eta\right)\right)\right\}
$$

when such a maximal $n$ exists, and -1 otherwise.

Let $N_{\alpha, A, A_{0}}=\max \left\{n_{k, l, a}:(k, l, a) \in \mathbb{Z}_{e} \times \mathbb{Z}_{e}^{m} \times A\right\}+1$. Take $n \geq N_{\alpha, A, A_{0}}$.

LEMMA 2.3. $o^{n}\left(\alpha / A A_{0}^{\geq n} \alpha(n)\right)=U$, where $U$ consists of the elements $\beta \in X^{m}$ such that $\beta(n)=\alpha(n)$ and for all $n^{\prime} \geq n, a \in A, \eta \in A_{0} \cap X_{n^{\prime}}$, $k \in \mathbb{Z}_{e}, l_{1}, \ldots, l_{m} \in \mathbb{Z}_{e}$ we have

$$
k\left|\left(\sum_{i=1}^{m} l_{i} \alpha_{i}\left(n^{\prime}\right)-a\left(n^{\prime}\right)-\eta\right) \Rightarrow k\right|\left(\sum_{i=1}^{m} l_{i} \beta_{i}\left(n^{\prime}\right)-a\left(n^{\prime}\right)-\eta\right) .
$$

Proof. $(\subseteq)$ is obvious.

$(\supseteq)$ The proof is an elaboration of the proof of Lemma 1.6. Take a $\beta \in U$. Let $k \geq 1, n \leq n_{1} \leq \cdots \leq n_{k},\left(a_{i j}\right)_{1 \leq i, j \leq k} \in M_{k \times k}\left(\mathbb{Z}_{e}\right),\left(b_{i j}\right)_{i \leq k, j \leq m} \in$ $M_{k \times m}\left(\mathbb{Z}_{e}\right), a=\left(a_{1}, \ldots, a_{k}\right) \in A^{k}$ and $\eta=\left(\eta_{1}, \ldots, \eta_{k}\right) \in\left(A_{0} \cap X_{n_{1}}\right) \times \cdots \times$ $\left(A_{0} \cap X_{n_{k}}\right)$.

We define the $\mathbb{Z}_{e}$-linear combinations $t_{i}(\bar{y}, \bar{x}, \bar{t}, \bar{z}), i=1, \ldots, k$, of variables $\bar{x}=\left(x_{1}, \ldots, x_{m}\right), \bar{y}=\left(y_{1}, \ldots, y_{k}\right), t=\left(t_{1}, \ldots, t_{k}\right)$ and $\bar{z}=\left(z_{1}, \ldots, z_{k}\right)$ by setting

$$
t_{i}(\bar{y}, \bar{x}, \bar{t}, \bar{z})=\sum_{j=1}^{k} a_{i j} y_{j}+\sum_{j=1}^{m} b_{i j} x_{j}+t_{i}+z_{i} .
$$

Now we choose $t_{i}^{\prime}(\bar{x}, \bar{t}, \bar{z}), i=1, \ldots, k$, as in the proof of Lemma 1.6.

By Lemma 2.2, to finish the proof we need to show that

$$
\begin{aligned}
&\left(\exists \bar{y} \in X^{k}\right)\left(\bigwedge_{i=1}^{k} t_{i}\left(\bar{y}\left(n_{i}\right), \alpha\left(n_{i}\right), a\left(n_{i}\right), \eta\right)=0\right) \\
& \Leftrightarrow\left(\exists \bar{y} \in X^{k}\right)\left(\bigwedge_{i=1}^{k} t_{i}\left(\bar{y}\left(n_{i}\right), \beta\left(n_{i}\right), a\left(n_{i}\right), \eta\right)=0\right) .
\end{aligned}
$$

$(\Rightarrow)$ Assume that for some $\bar{y} \in X^{k}$ we have

$$
\bigwedge_{i=1}^{k} t_{i}\left(\bar{y}\left(n_{i}\right), \alpha\left(n_{i}\right), a\left(n_{i}\right), \eta\right)=0 .
$$

As in the proof of Lemma 1.6 we get

$$
\bigwedge_{i=1}^{k} k_{i} \mid t_{i}^{\prime}(\alpha(n), a(n), \eta(n))
$$

By the choice of $n$ one can easily check that there is an $\eta^{\prime} \in\left(A_{0} \cap X_{n_{k}}\right)^{k}$ such that

$$
\bigwedge_{i=1}^{k} k_{i} \mid t_{i}^{\prime}\left(\alpha\left(n_{k}\right), a\left(n_{k}\right), \eta^{\prime}\left(n_{k}\right)\right) .
$$


From the assumption that $\beta \in U$ we see that

$$
\bigwedge_{i=1}^{k} k_{i} \mid t_{i}^{\prime}\left(\beta\left(n_{k}\right), a\left(n_{k}\right), \eta^{\prime}\left(n_{k}\right)\right) \text {. }
$$

The last two statements imply that we can choose $\bar{y}^{\prime}, \bar{y}^{\prime \prime} \in X^{k}$ so that

$$
\begin{aligned}
& \bigwedge_{i=1}^{k} t_{i}\left(\bar{y}^{\prime}\left(n_{i}\right), \alpha\left(n_{i}\right), a\left(n_{i}\right), \eta^{\prime}\left(n_{i}\right)\right)=0, \\
& \bigwedge_{i=1}^{k} t_{i}\left(\bar{y}^{\prime \prime}\left(n_{i}\right), \beta\left(n_{i}\right), a\left(n_{i}\right), \eta^{\prime}\left(n_{i}\right)\right)=0 .
\end{aligned}
$$

By $(*)$ and $(\mathrm{A})$ we infer that for $\bar{y}-\bar{y}^{\prime}=\left(y_{1}-y_{1}^{\prime}, \ldots, y_{k}-y_{k}^{\prime}\right)$ and $\theta=$ $\left(\eta_{1}-\eta^{\prime}\left(n_{1}\right), \ldots, \eta_{k}-\eta^{\prime}\left(n_{k}\right)\right)$ we have

$$
\bigwedge_{i=1}^{k} t_{i}\left(\left(\bar{y}-\bar{y}^{\prime}\right)\left(n_{i}\right), 0,0, \theta\right)=0 .
$$

Combining this with $(* *)$ shows that for $\bar{y}^{\prime \prime \prime}=\bar{y}^{\prime \prime}+\bar{y}-\bar{y}^{\prime}$ we have

$$
\bigwedge_{i=1}^{k} t_{i}\left(\bar{y}^{\prime \prime \prime}\left(n_{i}\right), \beta\left(n_{i}\right), a\left(n_{i}\right), \eta\right)=0
$$

The proof of $(\Leftarrow)$ is similar.

In the next corollary, $n$ is at least the maximum of the two numbers $N_{\alpha, A}$ and $N_{\alpha, A, A_{0}}$ defined before Lemmas 1.6 and 2.3, for $\alpha, A$ and $A_{0}$ as above.

COROLlarY 2.4. If we additionally assume that for each $i \geq n$, the set $A_{0} \cap X_{i}$ is contained in the kernel of the connecting map $f_{i, i-1}$, then $o^{n}\left(\alpha / A A_{0}^{\geq n} \alpha(n)\right)=o^{n}(\alpha / A \alpha(n))$.

Proof. $(\subseteq)$ is obvious.

() Take a $\beta \in o^{n}(\alpha / A \alpha(n))$. Suppose for contradiction that $\beta \notin$ $o^{n}\left(\alpha / A_{0}^{\geq n} A \alpha(n)\right)$. Lemma 2.3 shows that there are $a_{0} \in A_{0}$ (more precisely $a_{0} \in X_{n^{\prime}}$ for some $\left.n^{\prime}>n\right), a \in A$ and $k, l_{1}, \ldots, l_{m} \in \mathbb{Z}_{e}$ such that

$$
k \mid\left(\sum_{i=1}^{m} l_{i} \alpha_{i}\left(n^{\prime}\right)-a\left(n^{\prime}\right)-a_{0}\right) \text { and } k \nmid\left(\sum_{i=1}^{m} l_{i} \beta_{i}\left(n^{\prime}\right)-a\left(n^{\prime}\right)-a_{0}\right) .
$$

Since $a_{0}(n)=0$, it follows that $k \mid\left(\sum_{i=1}^{m} l_{i} \alpha_{i}(n)-a(n)\right)$. Hence, by the choice of $n$ we get $k \mid\left(\sum_{i=1}^{m} l_{i} \alpha_{i}-a\right)$. Consequently, $k \mid\left(\sum_{i=1}^{m} l_{i} \alpha_{i}\left(n^{\prime}\right)-a\left(n^{\prime}\right)\right)$, so $k \mid a_{0}$ and $k \mid\left(\sum_{i=1}^{m} l_{i} \beta_{i}\left(n^{\prime}\right)-a\left(n^{\prime}\right)\right)$. Finally, $k \mid\left(\sum_{i=1}^{m} l_{i} \beta_{i}\left(n^{\prime}\right)-a\left(n^{\prime}\right)-a_{0}\right)$.

Proposition 2.5. Assume that $X$ is the inverse limit of a system indexed by $\omega$ of finite abelian groups and $X$ has a finite exponent e. Let Aut $^{*}(X)$ be the standard structural group of $X$ and $A_{0}$ be an arbitrary subset of $X \cup \bigcup_{n \in \omega} X_{n}$. Then $\left(X, \operatorname{Aut}^{*}\left(X / A_{0}\right)\right)$ is m-normal and m-stable. 
Proof. Use Lemma 2.3 and proceed as in the proofs of Theorem 1.9 and Propositon 3.2.8 in [K1].

Proposition 2.6. Under the assumptions of Proposition 2.5, assume additionally that $A_{0} \subseteq \bigcup_{i \in \omega} X_{n}$ and for each $i \geq 1$, the set $A_{0} \cap X_{i}$ is contained in the kernel of the connecting map $f_{i, i-1}$. Then $\left(X, \operatorname{Aut}^{*}\left(X / A_{0}\right)\right)$ is small, m-normal and m-stable.

Proof. By Proposition 2.5 only smallness requires a proof. For two orbits $o_{1}$ and $o_{2}, o_{1} \subseteq_{o} o_{2}$ will mean that $o_{1}$ is open in $o_{2}$.

It is enough to show that for each $\alpha \in X$ and finite set $A \subseteq X$ we have $o\left(\alpha / A A_{0}\right) \subseteq_{o} o(\alpha / A)$. We can assume that for each $i \in \omega, A_{0} \cap X_{i}$ is a submodule of $X_{i}$.

Take an $n$ as in Corollary 2.4. Then

$$
o^{n}(\alpha / A \alpha(n))=o^{n}\left(\alpha / A A_{0}^{\geq n} \alpha(n)\right) .
$$

Since $o(\alpha / A \alpha(n)) \subseteq o^{n}(\alpha / A \alpha(n))$, we get

$$
o(\alpha / A \alpha(n)) \subseteq o^{n}\left(\alpha / A A_{0}^{\geq n} \alpha(n)\right) .
$$

On the other hand, by Remark 1.7 and Corollary 1.8 we see that

$$
o\left(\alpha / A A_{0} \alpha(n)\right) \subseteq_{o} o\left(\alpha / A A_{0}^{\geq n} \alpha(n)\right) \subseteq_{o} o^{n}\left(\alpha / A A_{0}^{\geq n} \alpha(n)\right) .
$$

Hence

$$
o\left(\alpha / A A_{0} \alpha(n)\right) \subseteq_{o} o(\alpha / A \alpha(n))
$$

Since we also have

$$
o\left(\alpha / A A_{0} \alpha(n)\right) \subseteq o\left(\alpha / A A_{0}\right) \quad \text { and } \quad o(\alpha / A \alpha(n)) \subseteq_{o} o(\alpha / A),
$$

we get $o\left(\alpha / A A_{0}\right) \subseteq_{o} o(\alpha / A)$.

Now we will make some final comments. First of all, Theorem 1.9 and Proposition 2.6 yield new classes of examples of small profinite groups. Next, recall the following question, which appears in [N1] and [N2]:

(*) Does there exist a small profinite group (structure) which is not $m$ normal?

Theorem 1.9 and Proposition 2.5 together with Remark 1.4 show that an example (if any) cannot be an abelian profinite group $X$ which is the inverse limit of a system $\left(X_{i}\right)_{i \in \omega}$ indexed by $\omega$, with the standard structural group or with the structural group being the pointwise stabilizer of a subset of $X \cup \bigcup_{n \in \omega} X_{n}$.

The next natural step in our investigations is to consider the profinite groups which are inverse limits of arbitrary countable systems (not necessarily indexed by $\omega$ ) of finite abelian groups of bounded exponent with the standard structural group. We could try to describe when such groups are small and to show that if such a group is small, then it is also $m$-normal 
(as in [K1, Theorem 4.2 and Proposition 4.3]). I think that the techniques of this paper together with some combinatorics should work here.

The situation is much more complicated when we consider abelian profinite groups (or even products of finite abelian groups) with arbitrary structural groups. Propositions 2.5 and 2.6 are partial results describing this situation. It seems to be very difficult to classify small abelian profinite groups with arbitrary structural groups. There is still a chance that there exists an abelian profinite group (or even a product of finite abelian groups) with some (rather complicated) structural group which is small, but not $m$-normal.

As at the end of Section 1, we mention that using many-sorted abelian structures we can give a new proof of the generalized description of orbits in products of finite abelian groups formulated in [K1, Lemma 3.1.2]. Namely, we can apply Lemma 2.2 (in fact, we use here the Baur-Garavaglia-Monk theorem for many-sorted abelian structures from example 5(i)) and some linear algebra (for the proof see [K3, Lemma 2.1.6]).

To end the paper I would like to explain that from some point of view, considering only abelian profinite groups is not a major restriction. We have to remember that our aim is to find examples of small profinite groups and that for such examples $m$-normality and $m$-stability are also considered.

In the case of a product $X=\prod_{i \in \omega} X_{i}$ of finite groups, Remark 4.3 from [N1] shows that while searching for small products of finite groups, we can assume that almost all $X_{i}$ 's are abelian. Then arguing as in the proof of Corollary 3.2.2 in [K1] we can assume that all $X_{i}$ 's are abelian.

In the case of arbitrary profinite groups we have the following fact proved by Wagner [Wa].

FACT 2.7. Each small $m$-stable profinite group contains an open abelian subgroup.

So searching for small and $m$-stable profinite groups, we can consider only profinite groups with an open abelian subgroup. When we have such a group $X$, then it is easy to see that $X$ is interpretable in its abelian canonical open subgroups and its smallness is equivalent to smallness of an arbitrary abelian canonical open subgroup (with some structural group). Assuming smallness and arguing as in the proof of Corollary 3.2.2 in [K1], we find that $m$-normality and $m$-stability of $X$ are also equivalent to $m$-normality and $m$-stability of an arbitrary abelian canonical open subgroup of $X$ (with the same structural group as above). So from the point of view of small and $m$-stable profinite groups (with arbitrary structural groups) we can consider only abelian profinite groups.

Without $m$-stability we do not have Wagner's theorem. Instead we have the open question whether each small profinite group contains an open abelian subgroup. It seems that considering non-abelian profinite groups 
(even with the standard structural group) is much more complicated and the techniques used in the description of orbits in abelian profinite groups do not work.

On the other hand, Newelski proved (see [N1] and [N3]) that any small $m$-normal profinite group has an open abelian subgroup. Hence, if we were able to find an example of a small profinite group without an open abelian subgroup (so also not $m$-stable), then we would get a positive answer to question $(*)$.

\section{References}

[A] M. Artin, Algebra, Prentice Hall, Englewood Cliffs, NJ, 1991.

[Ba] J. T. Baldwin, Fundamentals of Stability Theory, Springer, Heidelberg, 1988.

[Bu] S. Buechler, Essential Stability Theory, Springer, Berlin, 1996.

[F] E. Fisher, Abelian structures I, in: Lecture Notes in Math. 616, Springer, 1976, $270-322$.

[K1] K. Krupiński, Products of finite abelian groups as profinite groups, J. Algebra, to appear.

[K2] -, Profinite structures interpretable in fields, Ann. Pure Appl. Logic, submitted.

[K3] -, Profinite structures with various model theoretic properties, Ph.D. thesis, Wrocław, 2004.

[N1] L. Newelski, Small profinite groups, J. Symbolic Logic 66 (2001), 859-872.

[N2] -, Small profinite structures, Trans. Amer. Math. Soc. 354 (2002), 925-943.

[N3] -, M-gap conjecture and m-normal theories, Israel J. Math. 106 (1998), 285-311.

[PRZ] M. Prest, P. Rothmaler and M. Ziegler, Extensions of elementary duality, J. Pure Appl. Algebra 93 (1994), 33-56.

[RZ] L. Ribes and P. Zalesskii, Profinite Groups, Ergeb. Math. Grenzgeb. 40, Springer, 2000.

[Wa] F. Wagner, Small profinite m-stable groups, Fund. Math. 176 (2003), 181-191.

[We] V. Weispfenning, Quantifier elimination for modules, Arch. Math. Logik Grundlag. 25 (1985), 1-11.

[Z] M. Ziegler, Model theory of modules, Ann. Pure Appl. Logic 26 (1984), 149-213.

Mathematical Institute

University of Wrocław

Pl. Grunwaldzki 2/4

50-384 Wrocław, Poland

E-mail: kkrup@math.uni.wroc.pl
Institute of Mathematics Polish Academy of Sciences

Received 16 July 2004;

in revised form 9 February 2005 